\title{
Plasma and Lipids from Stored Packed Red Blood Cells Cause Acute Lung Injury in an Animal Model
}

\author{
Christopher C. Silliman, ${ }^{\star \star \pi}$ Norbert F. Voelkel, ${ }^{\ddagger}$ Jenny D. Allard, ${ }^{\ddagger}$ David J. Elzi," Rubin M. Tuder, ${ }^{\S}$ Jeffrey L. Johnson, \\ and Daniel R. Ambruso*ף

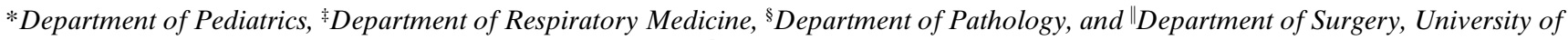 \\ Colorado School of Medicine, Denver, Colorado 80220; and " Bonfils Blood Center, Denver, Colorado 80220
}

\begin{abstract}
Transfusion-related acute lung injury (TRALI) is a serious complication of hemotherapy. During blood storage, lipids are generated and released into the plasma. In this study, the role of these lipids in TRALI was investigated using an isolated, perfused rat lung model. Rats were pretreated with endotoxin (LPS) or saline in vivo and the lungs were isolated, ventilated, and perfused with saline, or (a) $5 \%$ (vol/ vol) fresh human plasma, (b) plasma from stored blood from the day of isolation (D.0) or from the day of outdate (D.42), (c) lipid extracts from D.42 plasma, or (d) purified lysophosphatidylcholines. Lungs from saline or LPS-pretreated rats perfused with fresh (D.0) plasma showed no pulmonary damage as compared with saline perfused controls. LPS pretreatment/D.42 plasma perfusion caused acute lung injury (ALI) manifested by dramatic changes in both pulmonary artery pressure and edema. Incubation of LPS pre-tx rats with mibefradil, a $\mathrm{Ca}^{2+}$ channel blocker, or WEB 2170, a platelet-activating factor (PAF) receptor antagonist, inhibited ALI caused by D.42 plasma. Lung histology showed neutrophil sequestration without ALI with LPS pretreatment/saline or D.0 plasma perfusion, but ALI with LPS pretreatment/D.42 plasma perfusion, and inhibition of D.42 plasma induced ALI with WEB 2170 or mibefradil. A significant increase in leukotriene E4 was present in LPSpretreated/D.42 plasma-perfused lungs that was inhibited by WEB 2170 . Lastly, significant pulmonary edema was produced when lipid extracts of D.42 plasma or lysophosphatidylcholines were perfused into LPS-pretreated lungs. Lipids caused ALI without vasoconstriction, except at the highest dose employed. In conclusion, both plasma and lipids from stored blood produced pulmonary damage in a model of acute lung injury. TRALI, like the adult respiratory distress syndrome, may be the result of two insults: one derived from stored blood and the other from the clinical
\end{abstract}

Presented in part at The American Society for Hematology National Meeting, Orlando, FL, December 1996.

Address correspondence to Daniel R. Ambruso, M.D., Bonfils Blood Center, 717 Yosemite Circle, Denver, CO 80220. Phone: 303363-2241; FAX: 303-340-2616; E-mail: daniel.ambruso@uchsc.edu

Received for publication 26 September 1997 and accepted in revised form 27 January 1998.

J. Clin. Invest.

(C) The American Society for Clinical Investigation, Inc. 0021-9738/98/04/1458/10 \$2.00

Volume 101, Number 7, April 1998, 1458-1467

http://www.jci.org condition of the patient. (J. Clin. Invest. 1998. 101:14581467.) Key words: rat lungs - lysophosphatidylcholines • mibefradil • WEB 2170 • leukotriene E4

\section{Introduction}

Although critical in host defense, the neutrophil has been implicated as a mediator of tissue destruction in a wide variety of inflammatory diseases ranging from rheumatoid arthritis to acute lung injury (ALI) ${ }^{1}$ including the adult respiratory distress syndrome (ARDS) (1-4). Neutrophils play a central role in the pathogenesis of ALI in human patients as evidenced by: (a) the widespread infiltration of the lung with hyperactive neutrophils (PMNs); $(b)$ the presence of potent PMN chemoattractants in the bronchoalveolar lavage fluid; and $(c)$ the clinical deterioration of neutropenic patients with ALI upon neutrophil recovery $(3,5-8)$.

Neutrophils may be primed or activated. Distinct from activation, neutrophil priming is a process that augments responses to subsequent stimuli (9-11). Priming not only maximizes the microbicidal function of neutrophils in response to subsequent stimuli, but also causes a direct increase in surface expression of the $\beta_{2}$-integrin adhesion molecules leading to increased neutrophil adherence especially to vascular endothelium (12-14). Upon activation of primed neutrophils, maximal amounts of toxic oxygen metabolites and nonoxidative proteins from the azurophilic and specific granules, are released into the phagolysozome or at points of firm adhesion mediated by the $\beta_{2}$-integrins and their ligands on endothelium and other cells (12-16).

Extensive investigation of animal models has lead to the conclusion that ALI is the result of at least two independent events mediated by separate agents $(17,18)$. The first agent primes neutrophils and activates endothelium resulting in indiscriminate adherence of these neutrophils to the activated pulmonary endothelium via increased surface expression of adhesion molecules $(13,17,18)$. A subsequent, second agonist then activates these adherent neutrophils causing release of cytotoxic products, endothelial damage, and capillary leak (13, 17, 18).

Transfusion of blood components has been temporally associated with the development of ALI and the clinical syn-

1. Abbreviations used in this paper: AII, angiotensin II; ALI, acute lung injury; ARDS, the adult respiratory distress syndrome; D.0, plasma isolated from packed red blood cell plasma on the day of product isolation; D.42, plasma isolated from packed red blood cells on the day of outdate, day 42 of storage, the last day the unit may be transfused; fMLP, formyl-Met-Leu-Phe; LPS, endotoxin; LTE4, leukotriene E4; lyso-PCs, lysophosphatidylcholines; $\mathrm{O}_{2}{ }^{-}$, superoxide anion; PA, pulmonary artery; PAF, platelet-activating factor; PMN, neutrophil; PRBCs, packed red blood cells; TRALI, transfusionrelated acute lung injury. 
drome termed transfusion-related acute lung injury (TRALI) $(19,20)$. Previously, TRALI has been related to leukocyte antibodies causing complement activation, pulmonary sequestration of granulocytes, and activation of these adherent phagocytes resulting in lung injury (19). TRALI is clinically similar if not identical to ALI/ARDS (19-21). Recently, the list of agents implicated in the pathogenesis of TRALI has grown longer to include neutrophil priming agents that are generated during routine blood storage (22). Furthermore, TRALI has been postulated to occur as the result of two sequential insults: first, an underlying clinical condition was present that may have predisposed the patients to acute lung injury, such as recent surgery, cytokine treatment, massive blood transfusion, and active infection, and second, transfusion of active lipid compounds in stored blood (22). Based upon the two event model of ALI involving neutrophil priming and activation, an animal model of TRALI was developed $(17,18)$. LPS was used as the first insult to attract neutrophils to the pulmonary circulation and to prime neutrophils and activate the pulmonary endothelium. The ability of plasma and lipids from cellular, stored blood components to act as a second agonist in the production of ALI was evaluated during the perfusion phase. The results indicate that lipids, generated in the plasma fraction during blood storage, may serve as one of two events required for pulmonary injury.

\section{Methods}

Materials. Formyl-Met-Leu-Phe (fMLP), platelet activating factor (PAF), cytochrome c, endotoxin from Salmonella enteritides, essentially fatty acid free human albumin, superoxide dismutase, 1-ostearoyl-sn-glycero-3-phosphocholine, 1-o-palmitoyl-sn-glycero-3-phosphocholine, 1-o-oleyl-sn-glycero-3-phosphocholine, and 1-o-hexadecyl-sn-glycero-3-phosphocholine $\left(\mathrm{C}_{16}\right.$ lyso-PAF), and angiotensin II (AII) were purchased from Sigma Chemical Co. (St. Louis, MO). Male Sprague Dawley rats (300 g) were obtained from Harlan Sprague Dawley Inc. (Indianapolis, IN) and quarantined for $7 \mathrm{~d}$ before initiation of any experimentation. Plastic microplates manufactured by Nunc Inc. (Naperville, IL) were purchased from Intermountain Scientific Inc. (Bountiful, UT). WEB 2170 was the kind gift of Boehringer Ingelheim Pharmaceuticals Inc. (Ridgefield, CT). Mibefradil was a gift from Dr. F.P. Clozel (Hoffman Laroche, Basel, Switzerland). One-step animal nycodenz-based density gradient: density $1.077 \mathrm{~g} / \mathrm{ml}$ and osmolarity of $265 \mathrm{mosoms} / \mathrm{liter}$, was obtained from Accurate Chemical and Scientific Co. (Westbury, NY). Hetastarch $6 \%$ in $0.9 \%$ saline was purchased from McGaw, Inc. (Irvine, CA).

Plasma preparation from stored packed red blood cells. For the initial experiments, healthy adult volunteers donated $1 \mathrm{U}$ of whole blood after informed consent had been obtained according to the $\mathrm{Hu}$ man Subjects Committee at the University of Colorado School of Medicine. Approximately $450 \mathrm{ml}$ of whole blood were added to $67 \mathrm{ml}$ of anticoagulant preservative solution (citrate-phosphate-dextroseadenine). The units were separated by standard centrifugation techniques into components: packed red blood cells (PRBCs), platelets, and plasma. The PRBCs were stored at $4^{\circ} \mathrm{C}$ according to the American Association of Blood Banks standards (23). Samples, 35-50 ml, were obtained on the day of collection and at outdate, the last day the unit may be transfused, through sterile couplers using sterile technique. The samples were centrifuged at 5,000 $\mathrm{g}$ for $7 \mathrm{~min}$ to remove cells followed by a second centrifugation step at $12,500 \mathrm{~g}$ for $5 \mathrm{~min}$ to remove acellular debris. The supernatants were removed, and individual aliquots were stored at $-70^{\circ} \mathrm{C}$.

Before addition to the perfusate of the isolated rat lungs, the plasma samples were heated at $56^{\circ} \mathrm{C}$ for $45 \mathrm{~min}$ to eliminate the bioactivity of fibrinogen and complement. After the initial experiments that investigated pulmonary damage caused by paired PRBC plasma samples isolated from packed red blood cell plasma on the day of product isolation (D.0) and plasma isolated from packed red blood cells on the day of outdate, day 42 of storage, the last day the unit may be transfused (D.42) of the identical units, plasma samples from a number of PRBC units were collected on D.42 and pooled for use in a number of the following studies. As additional controls, adeninecitrate-dextrose anticoagulated whole blood was collected by venipuncture from healthy human donors, plasma was separated by standard techniques (23) and used for control experiments.

The isolated perfused rat lung. Healthy, adult male, Sprague Dawley rats $(300 \mathrm{~g})$ raised on a regular diet were weighed and injected intraperitoneally with LPS $(2 \mathrm{mg} / \mathrm{kg}$ ) or Earls buffered salt solution, $\mathrm{pH}$ 7.4, (saline) and observed for $2 \mathrm{~h}$. After this incubation period, the rats were anesthetized with sodium pentobarbital $(80 \mathrm{mg} / \mathrm{kg}$, i.p.), and a median sternotomy was performed so that the lungs could be isolated, perfused with saline (constant rate of $0.03 \mathrm{ml}$ per gram per minute), and mechanically ventilated as described previously (24). To test the hypothesis that plasma from stored blood causes ALI, the lungs were perfused with saline (control), $5 \%(\mathrm{vol} / \mathrm{vol})$ heat treated fresh human plasma, or $5 \%$ of the plasma fraction from packed red blood cells from D.0 or D.42 of storage. Pulmonary artery pressure $(\mathrm{mmHg})$ was measured as described previously (24). After perfusion, the rat lungs were weighed, and then fixed for histologic examination. Preliminary studies delineated the percentage of D.42 PRBC plasma to be used in these experiments. Lower percentages of D.42 PRBC plasma in the perfusate $(0.5-2.0 \%)$ yielded minimal, direct increases in pulmonary artery pressures in lungs from LPS-pretreated animals, 1-4 $\mathrm{mmHg}$, whereas higher percentages (5-10\%) demonstrated direct increases in pulmonary artery pressure from $14-35 \mathrm{mmHg}$. A dose of $5 \%$ D. 42 PRBC plasma was chosen to explore the two event model of lung injury. Additional experiments examined whether perfusion with lipid extracts of the plasma samples (D.0 or D.42 from PRBCs) or mixtures of purified lysophosphatidylcholines $(5-22.5 \mu \mathrm{M})$, the major active lipid species generated during routine blood storage, also cause ALI (25).

Lipid extractions. Lipids were extracted from plasma samples using a 1:1:1 methanol-2.0\% acetic acid/chloroform/water extraction solution (25). The samples were centrifuged at $200 \mathrm{~g}$ for $10 \mathrm{~min}$ at $4^{\circ} \mathrm{C}$. to aid in separation of the phases. The chloroform soluble phase was removed and dried. The dried lipids were solubilized in $1.25 \%$ essentially fatty acid free human albumin in a volume identical to that of the plasma extracted for perfusion into the isolated rat lungs.

Leukotriene E4 (LTE4) measurement in perfusate. The perfusate was measured before and $30 \mathrm{~min}$ after the addition of heat-treated, D.42 PRBC plasma. LTE4 was quantitated using an enzyme linked immunosorbent assay as reported previously (26).

Histology. Lungs were inflated, fixed in $4 \%$ formalin, paraffin embedded, and sectioned. Sections were stained with hematoxylin and eosin and examined by light microscopy.

Neutrophil isolation and oxidase priming. PMNs were isolated from whole blood obtained from healthy, male Sprague Dawley rats weighing 300-400 $\mathrm{g}$ using a modification of the hespan exchange transfusion technique (27). Rats were anesthetized with $100 \mathrm{mg} / \mathrm{kg}$ of ketamine and $2.5 \mathrm{mg} / \mathrm{kg}$ acepromazine injected into the peritoneal cavity. A midline laparotomy was performed in a sterile fashion, and the inferior vena cava was cannulated. Serial $2.5 \mathrm{ml}$ aliquots of whole blood were drawn followed by bolus infusion of $2.5 \mathrm{ml}$ of $6 \%$ hetastarch $0.9 \%$ saline through a three-way stopcock until the blood volume had been completely exchanged for Hespan-saline. The total procedure lasted $\sim 10 \mathrm{~min}$, and all animals maintained spontaneous respirations and circulatory integrity. The rat blood was mixed 1:1 (vol/vol) with $6 \%$ hetastarch $0.9 \%$ saline and the red blood cells allowed to sediment for $35 \mathrm{~min}$. The neutrophil rich upper layer was removed and the cells pelleted for $10 \mathrm{~min}$ at $200 \mathrm{~g}$. The pellet was resuspended in PBS, $\mathrm{pH} 7.4$, and layered over $5 \mathrm{ml}$ of 1-Step Animal nycodenz gradient $(1.077 \mathrm{~g} / \mathrm{cc}$ density, $265 \mathrm{mosom} / \mathrm{liter})$ and centrifuged at $600 \mathrm{~g}$ for $20 \mathrm{~min}$. Contaminating red blood cells were re- 
moved by hypotonic lysis, and the neutrophils were resuspended in Krebs-Ringer phosphate buffer, $\mathrm{pH} 7.35$, with $2 \%$ dextrose. The rat neutrophils were $>95 \%$ pure as determined by Wright's staining of smears and examination by light microscopy. Viability was $>99 \%$ by Trypan Blue exclusion.

Priming assays on isolated rat neutrophils were completed at $37^{\circ} \mathrm{C}$ in duplicate with a separate superoxide dismutase control. Briefly, rat neutrophils were primed for $3 \mathrm{~min}$ with buffer, a mixture of lyso-PCs $(5 \mu \mathrm{M})$, or $2 \mu \mathrm{M}$ PAF. The respiratory burst was then initiated by the addition of $1 \mu \mathrm{M}$ fMLP. The maximal rate of superoxide anion $\left(\mathrm{O}_{2}{ }^{-}\right)$ production was measured as the superoxide dismutase inhibitable reduction of cytochrome c at $550 \mathrm{~nm}$ of light in a Molecular Dynamics (Sunnyvale, CA) Microplate Reader as described (25).

Data analysis. The means, SDs, and SEMs were calculated for each experimental and control group by standard methods. Statistical differences $(P<0.05)$ among groups were measured by a paired ANOVA for repeated measurements or an independent ANOVA for comparison of independent groups both followed by the Student Newman Keuls test for multiple comparisons.

\section{Results}

Effects of plasma from stored PRBCs on pulmonary artery $(P A)$ pressure. Isolated lungs from rats intraperitoneally injected with saline in vivo (saline pretreatment) and perfused with fresh plasma or D.0 PRBC plasma did not show any demonstrable increases in PA pressure compared with control lungs from rats pretreated with saline and perfused with saline (Fig. 1, $A$ and $B$ ). In addition, there was no augmentation of the vasoconstrictive response to a hypoxic challenge $\left(\mathrm{FiO}_{2}=\right.$ $3 \%$ ) or the response after bolus injection of $1 \mu \mathrm{g}$ of AII. Moreover, pretreatment of rats with an intraperitoneal endotoxin (LPS) injection in vivo, before lung isolation, (LPS pretreatment) followed by perfusion with fresh plasma or D.0 PRBC plasma did not cause a significant increase in PA pressures nor an augmentation of the vasoconstrictive responses to either hypoxia or AII (Fig. $1 C$; Fig. 2). In contrast, when lungs from saline pretreated rats were perfused with D.42 PRBC plasma, a small but reproducible increase in PA pressure was noted (Fig. $1 \mathrm{D})$. It is important to note that all reported changes in PA pressures occurred directly in response to the addition of plasma or lipids to the perfusate (Fig. 1, arrows) and the response was only considered over the initial $10 \mathrm{~min}$ of the perfusion, well before the addition of AII. In addition, an augmentation of both the hypoxic and AII responses as compared with either lungs perfused with saline, fresh plasma, or D.0 PRBC plasma was also observed. Moreover, after the bolus injection of ATII, there was a sustained elevation in the baseline PA pressures (Fig. $1 \mathrm{D}$ ). This sustained elevation in PA pressures occurred in all five lung preparations from saline pretreated animals perfused with D.42 PRBC plasma, from five separate PRBC units, after the initial dose of ATII. Despite this persistent elevation of PA pressure, the vasoconstrictive response to both ATII and hypoxia was augmented as compared with the control lungs or to D.0 or fresh plasma perfused rat lungs from animals pretreated with LPS (Fig. 1, $A-D$ ). Strikingly, LPS pretreatment followed by perfusion of the isolated lungs with $5 \%$ D. 42 PRBC plasma resulted in a large, rapid pressor response after the addition of the plasma to the perfusate (Fig. 1 $E$; Fig. 2) $(P<0.05)$. Similar to the lungs from saline pretreated rats perfused with D.42 PRBC plasma, the PA pressure was persistently elevated after the initial dose of ATII. However, the vasoconstrictive responses to both ATII and hypoxia were
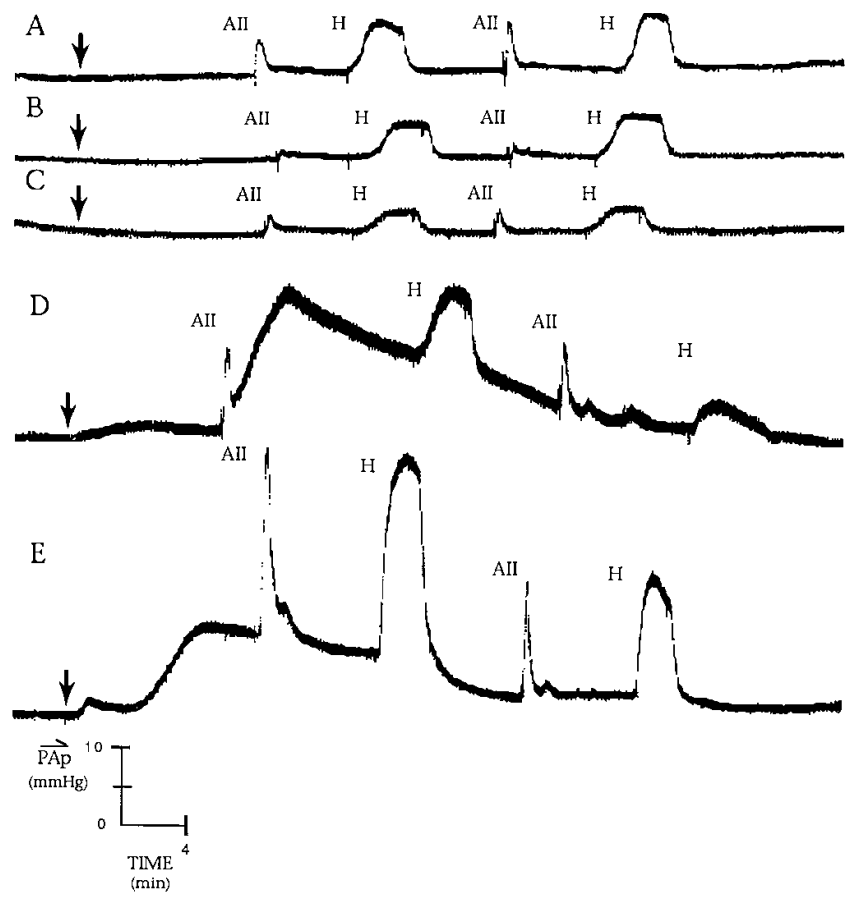

Figure 1. Pulmonary artery pressure tracings obtained from isolated perfused rat lungs at constant flow. In Hanks' buffered saline-pretreated, saline-perfused lungs $(A)$ alveolar hypoxia $(H, 3 \%)$ or a bolus injection of angiotensin II $(A I I, 1 \mu \mathrm{g})$ caused physiologic vasoconstriction. $(B)$ Perfusion of isolated lungs from a saline-pretreated animal with D.0 PRBC plasma (5\%, vol/vol) does not demonstrate direct vasoconstriction nor an augmented response to hypoxia or AII. (C) Perfusion of lungs from an LPS-pretreated rat with D.0 PRBC plasma did not demonstrate direct changes in PA pressures nor augmentation of the vasoconstrictive response to hypoxia or AII infusion. $(D)$ Perfusion of lungs from a buffer- pretreated animal perfused with D.42 PRBC plasma (5\%, vol/vol) shows a direct vasoconstrictive response with augmentation of the normal pressor response to hypoxia and AII infusion. (E) Perfusion of D.42 PRBC plasma into lungs from an LPS-pretreated rat demonstrate a marked, direct increase in PA pressures as well as augmentation of the pressor responses to both hypoxia and AII infusion.

still potentiated compared with control lungs or to saline and LPS-pretreated lungs perfused with D.0 PRBC plasma.

Addition of $50 \mu \mathrm{M}$ WEB 2170, a platelet activating factor receptor antagonist (28-30), to the perfusate of the lungs from LPS-pretreated rats 20 min before perfusion with D.42 PRBC plasma, resulted in significant inhibition of the increased PA pressure (91 $\pm 4 \%$, mean \pm SEM, $n=5$ ) (Fig. 2). WEB 2170 also caused a diminution of the augmented vasoconstrictive responses to both hypoxia and AII (results not shown). Analogous incubation of rat lungs with mibefradil, a calcium channel antagonist $(31,32)$, inhibited the increase in PA pressure $(45 \pm 12 \%, n=5)$ in LPS-pretreated rats perfused with D.42 PRBC plasma but to a lesser degree than the inhibition observed with WEB 2170 (Fig. 2).

Effects of plasma from stored PRBCs on pulmonary edema. To quantitate the degree of pulmonary edema, the ratio of wet lung weight to total body weight was obtained. Pretreatment of rats with saline followed by perfusion of the lungs with saline, fresh plasma, D.0 PRBC plasma, or D.42 PRBC 


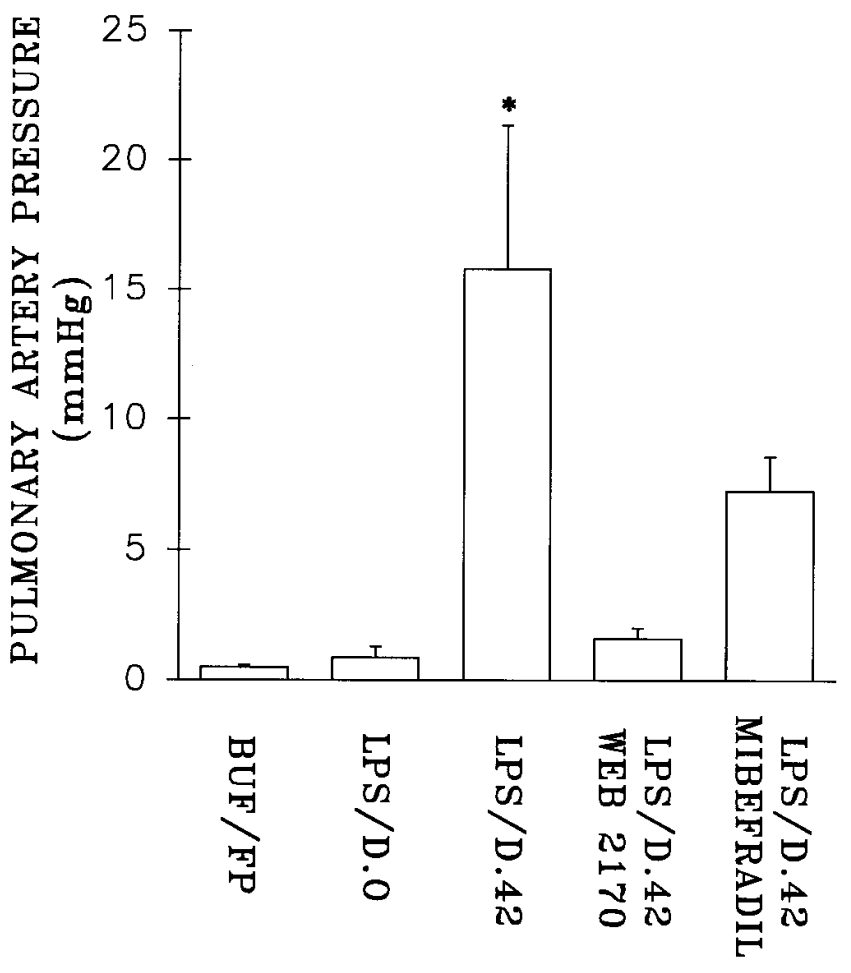

Figure 2. Pulmonary artery pressure as a function of pretreatment and perfusion groups in isolated, perfused lungs from rats. Perfusion of D.42 PRBC plasma caused a significant increase in pulmonary artery pressure compared with fresh plasma $(F P)$ or D.0 PRBC plasma perfusion in lungs from LPS-pretreated animals $(P=0.02, n=5)$. Preincubation (10 $\mathrm{min}$ ) of the isolated perfused lungs with $50 \mu \mathrm{M}$ WEB $2170(91 \pm 4 \%$, mean \pm SEM, $n=5)$ or $10 \mu \mathrm{M}$ mibefradil $(45 \pm 12 \%$, $n=4)$ inhibited these vasoconstrictive responses in LPS-pretreated D.42 PRBC plasma-perfused rats.

plasma did not affect the lung weight to total body weight ratio (results not shown). Moreover, pretreatment of rats with LPS followed by lung isolation and saline perfusion did not demonstrate a significant increase in lung weight (results not shown). No increase in lung weight was detected in the LPS-pretreated rats perfused with fresh plasma or plasma from PRBCs on the day of collection (Fig. 3). However, when LPS pretreatment was followed by perfusion with D.42 PRBC plasma, a significant increase in lung weight was observed as compared with the fresh plasma or D.0 PRBC plasma $(P<0.05)$. Addition of WEB 2170 or mibefradil to the perfusate of LPS-pretreated rats before perfusion with D.42 PRBC plasma reduced pulmonary edema $(P<0.05)$ (Fig. 3). The inhibition of edema formation by WEB 2170 and mibefradil was $55 \pm 16 \%(n=5)$ and $53 \pm 7 \%(n=4)$, respectively.

Histologic examination of the rat lungs. Lungs from salinepretreated rats perfused with fresh plasma, D.0 PRBC plasma, or D.42 PRBC plasma, had normal pulmonary architecture with sparse numbers of inflammatory leukocytes especially in the interstitium (Fig. 4, $A$ and $B$ ). Conversely, LPS pre-treatment of the rats followed by perfusion with fresh plasma, D.0 PRBC plasma or saline resulted in leukocyte sequestration throughout the small pulmonary arterioles and capillaries without evidence of septal or interstitial damage (Fig. $4 C$ ). In marked contrast to these conditions, LPS pretreatment fol- lowed by lung isolation and perfusion with D.42 PRBC plasma demonstrated perivascular edema (Fig. $4 D$ ) and both septal inflammation and edema with marked neutrophil infiltration (Fig. 4, $D$ and $E$ ). Pre-incubation with either WEB 2170 (Fig. $4 F$ ) or mibefradil (results not shown) before perfusion with D.42 PRBC plasma did not alter neutrophil sequestration, but decreased the observed pulmonary intravascular and septal injury, especially compared with Fig. 4, $D$ and $E$.

Measurement of LTE4 from pulmonary perfusate. Levels $(\mathrm{pg} / \mathrm{ml})$ of LTE4 were measured in the lung perfusate of LPSpretreated rats before and after addition of the D.42 PRBC plasma. Perfusion with D.42 PRBC plasma in lungs from animals pretreated with LPS showed a significant 3.0 \pm 1.0 -fold increase in the levels of LTE4 as compared with lungs after LPS pre-treatment and saline perfusion $(P=0.03, n=4)$ (Table I). The increase in LTE4 concentration was inhibited by preincubation of LPS-pretreated lungs with $50 \mu \mathrm{M}$ WEB 2170 before perfusion with D.42 PRBC plasma. Analogous experiments demonstrated that preincubation with $10 \mu \mathrm{M}$ mibefradil also decreased the generation of LTE4 after 5\% D.42 PRBC perfusion of the lungs from LPS-pretreated animals but to a milder degree than WEB 2170 (64 $14 \%$ versus $76 \pm 11 \%)$. Lastly, there was no increase in LTE4 levels in lungs from LPS-pretreated rats perfused with buffer or either fresh plasma or D.0 PRBC plasma.

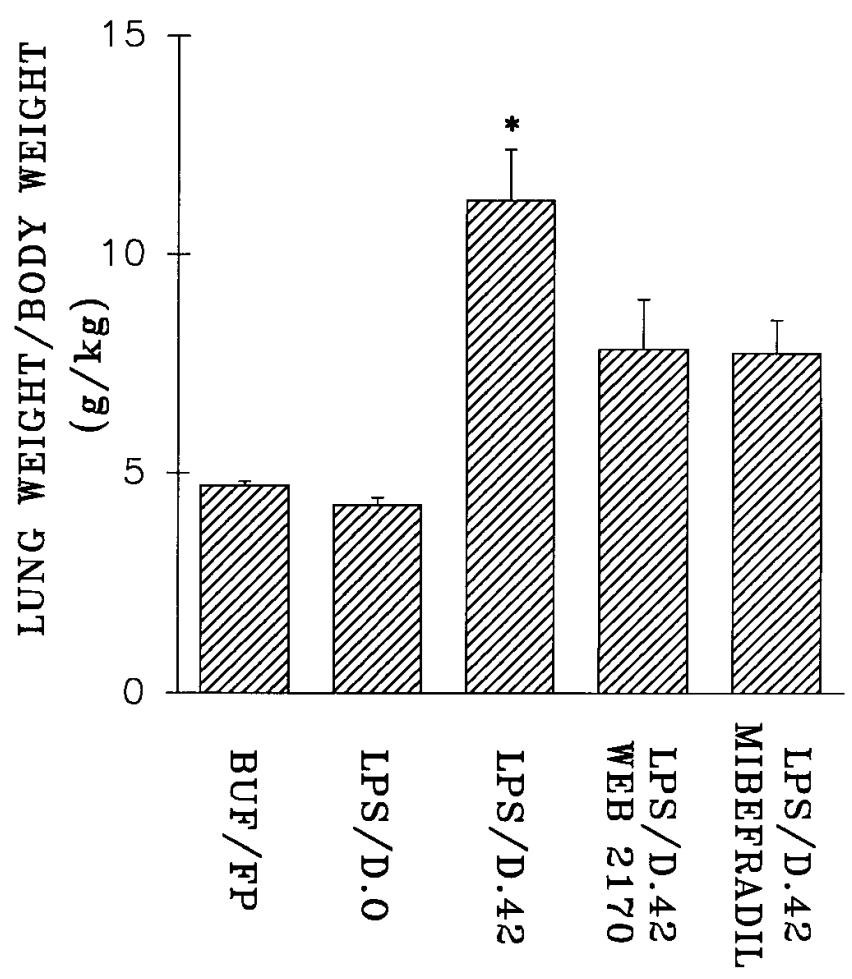

Figure 3. Changes in the ratio of wet lung weight to total body weight $(\mathrm{g} / \mathrm{kg})$, a measure of pulmonary edema, as a function of pretreatment and perfusion groups in isolated perfused rat lungs. Perfusion of D.42 PRBC plasma into the lungs from LPS-pretreated rats demonstrated a significant increase in pulmonary edema as compared with LPSpretreated, fresh plasma $(F P)$, or D.0 PRBC plasma-perfused lungs $(P=0.01)$. Preincubation with $50 \mu \mathrm{M}$ WEB 2170 or $10 \mu \mathrm{M}$ mibefradil inhibited these increases in pulmonary edema by $55 \pm 16 \%(n=5)$ and $53 \pm 7 \%(n=4)$, respectively. 

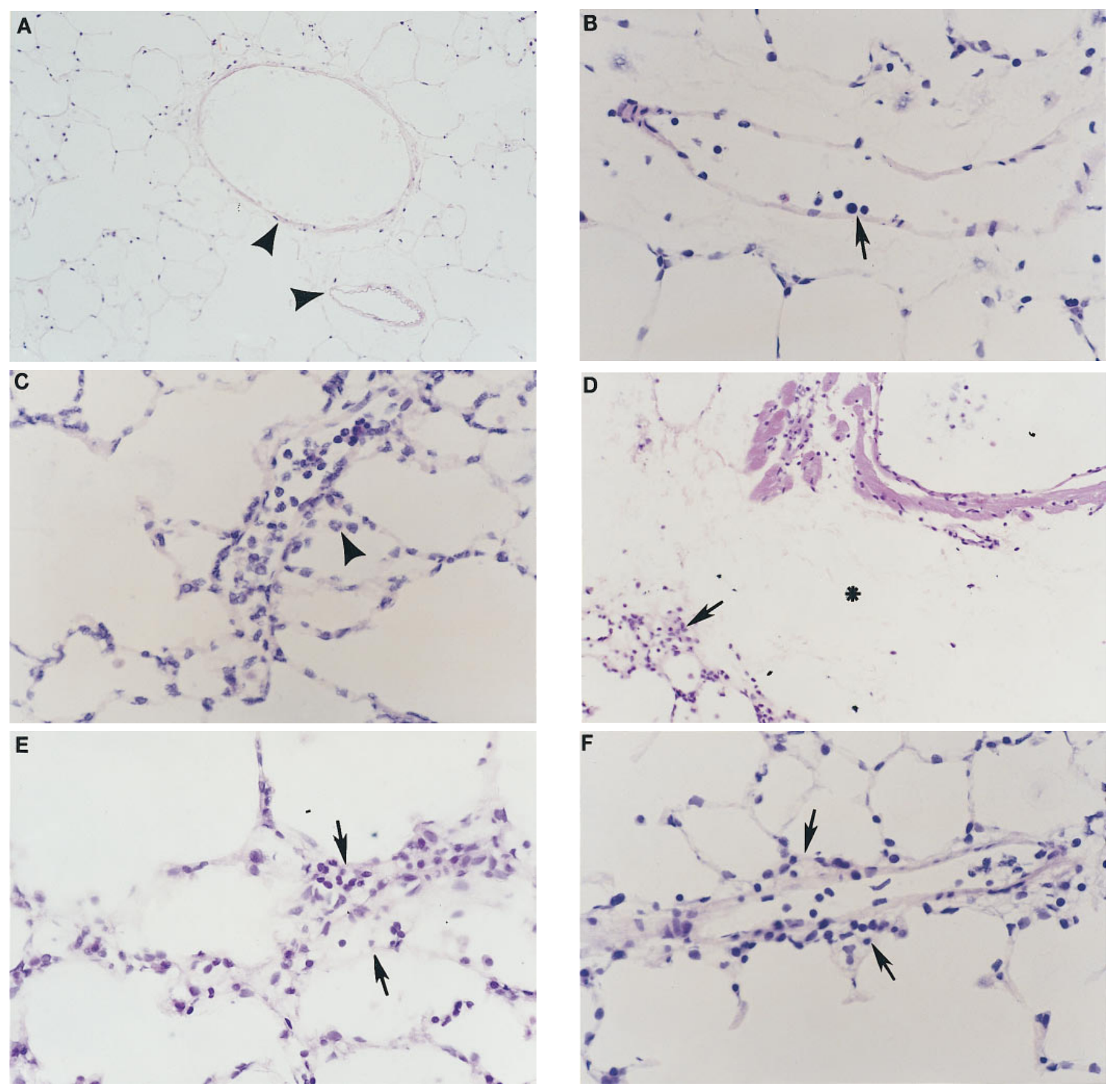

Figure 4. Histologic examination of sections of isolated perfused rat lungs from different treatment groups. $(A)$ Lungs from saline-pretreated perfused with saline show normal pulmonary histology. $(B)$ The lungs from saline-pretreated D.0 PRBC plasma (5\%, vol/vol)-perfused animals show normal lung histology. $(C)$ The lungs from LPS-pretreated animals perfused with D.0 PRBC plasma show PMN sequestration throughout the capillaries without evidence of pulmonary damage. $(D)$ Lungs from rats pretreated with LPS, perfused with D.42 PRBC plasma (5\%, vol/ vol) demonstrate widespread pulmonary damage with perivascular cuffing, hyaline membranes and edema formation. $(E)$ Lungs from LPS-pretreated animals preincubated with $50 \mu \mathrm{M}$ WEB 2170 before perfusion with D.42 PRBC plasma demonstrate neutrophil sequestration without evidence of pulmonary damage. $(F)$ Lungs from LPS-pretreated animals preincubated with $10 \mu \mathrm{M}$ mibefradil also demonstrate neutrophil sequestration without evidence of ALI similar to $E$.

Changes in PA pressure and pulmonary edema in response to gross lipid extracts and purified lipids from PRBC plasma. A number of biologically active compounds may be generated during blood storage including lipids that are chloroform extractable and proteins and peptides that are not. Therefore, the lipids from the D.42 PRBC plasma samples were extracted to examine whether the lipid priming activity generated during blood storage was capable of producing pulmonary damage in the lungs from LPS-pretreated animals. Perfusion of the lipid extracts of D.42 PRBC plasma into lungs from LPS-pretreated rats produced significant amounts of pulmonary edema as compared with control rats pretreated with LPS and perfused 
Table I. Measurement of Leukotriene E4 in Lung Perfusate from Intraperitoneal LPS Pretreated Rats

\begin{tabular}{lcc}
\hline & $\begin{array}{c}\text { Before perfusion } \\
\text { with D.42 PRBC plasma }\end{array}$ & $\begin{array}{c}\text { After perfusion } \\
\text { with D.42 PRBC plasma }\end{array}$ \\
\hline $\begin{array}{l}\text { Saline preincubation } \\
50 \mu \text { M WEB 2170 } \\
\text { preincubation }\end{array}$ & $196.8 \pm 55.7$ & $590.2 \pm 106.1^{*}$ \\
& $249.7 \pm 15.7$ & $417.7 \pm 89.3$
\end{tabular}

LTE4 was measured in $\mathrm{pg} / \mathrm{ml}$ as described (26). The rat lungs were perfused with saline or WEB 217010 min before perfusion with D.42 PRBC plasma. *Statistical significance as compared to saline-perfused controls from intraperitoneal LPS pretreated rats.

with D.0 PRBC plasma. However, no significant differences in PA pressures were elicited by perfusion of the lungs from intraperitoneal LPS-pretreated rats with lipid extracts of D.42 PRBC plasma (Fig. 5). In addition, perfusion of extracts of D.0 PRBC plasma into the lungs of LPS-pretreated rats also did not demonstrate significant pulmonary edema as compared with the LPS-pretreated, D.0 PRBC plasma-perfused controls.

Previous data demonstrated that lysophosphatidylcholines are the active lipid compounds found in whole blood, PRBCs, and platelet concentrates $(25,33)$. Moreover, both the molecular structures and relative concentrations at PRBC outdate (D.42) have been reported previously (25). Purified lyso-PCs

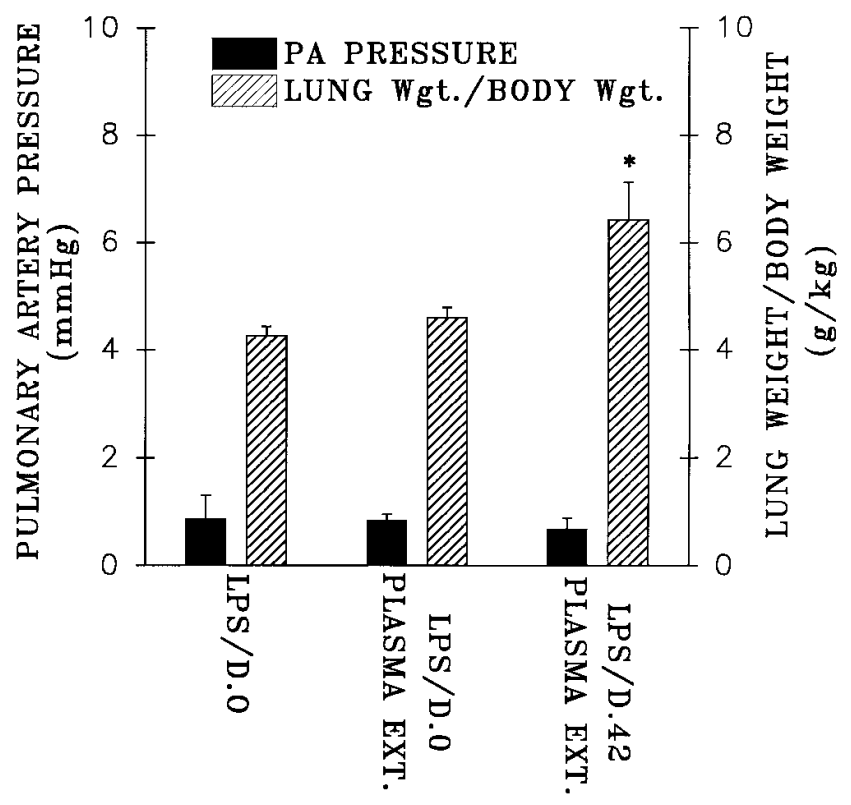

Figure 5. Changes in both pulmonary artery pressure $(\mathrm{mmHg}$, left ordinate) and the ratio of wet lung weight to body weight $(\mathrm{g} / \mathrm{kg}$, right ordinate) as a function of the different perfusates in isolated perfused lungs from LPS-pretreated rats. There was no difference in vasoconstrictive responses in lungs from LPS-pretreated animals after D.42 PRBC plasma perfusion as compared with lungs from LPS-pretreated rats perfused with D.0 PRBC plasma $(n=5)$. However, perfusion with D. 42 PRBC plasma in lungs from LPS-pretreated animals did cause a significant increase in pulmonary edema as compared perfusion of D.0 PRBC plasma into lungs from LPS-pretreated animals $(P=0.03, n=5)$.

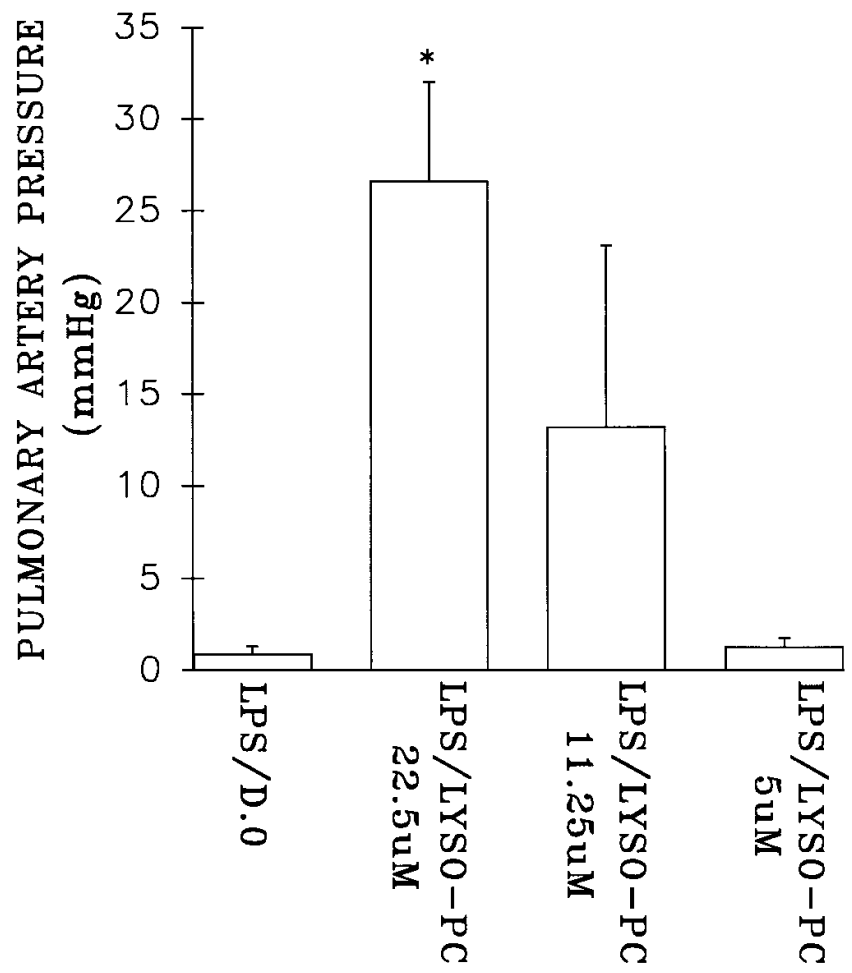

Figure 6. Changes in pulmonary artery pressure as a function of the concentration of lysophosphatidylcholines in the perfusate compared with perfusion with 5\% D.0 PRBC plasma in lungs from rats pretreated with LPS. Lyso-PCs at the highest dose $(22.5 \mu M)$ caused a significant increase in PA pressures as compared with LPS-pretreated D.0 PRBC plasma-perfused controls $(P=0.04, n=6)$ while the lower doses of lyso-PCs in the lungs from LPS-pretreated rats did not demonstrate significant increases in PA pressure as compared with the LPS-pretreated D.0 PRBC plasma-perfused animals.

from commercial suppliers were obtained and solubilized with $1.25 \%$ human albumin. LPS-pretreated rats perfused with a mixture of lyso-PCs demonstrated a significant and dosedependent increase in PA pressures (Fig. 6). Significant pulmonary vasoconstriction was seen with a dose of $22.5 \mu \mathrm{M}$ lysoPCs as compared with LPS-pretreated, saline-perfused animals, and this increase in PA pressures was inhibited by preincubation with $50 \mu \mathrm{M}$ WEB 2170 by $80+7 \%(P=0.03)$. Conversely, lesser concentrations of lyso-PCs, $11.25 \mu \mathrm{M}$ and $5 \mu \mathrm{M}$, perfused into lungs from LPS-pretreated rats did not cause significant pulmonary vasoconstriction as compared with LPSpretreated, saline-perfused controls. Perfusion of isolated lungs from LPS-pretreated animals with various concentrations of lyso-PCs caused pulmonary edema for all concentrations of lyso-PCs tested (5-22.5 $\mu \mathrm{M})$ (Fig. 7). The increases in edema formation appeared to have been dose dependent with maximal lung injury occurring with the highest lyso-PC concentration used. Moreover, all lyso-PC doses elicited significant pulmonary edema as compared with lungs from LPS-pretreated animals perfused with saline. WEB 2170 preincubation demonstrated modest inhibition, $29 \pm 10 \%$ of pulmonary edema formation in response to the $22.5 \mu \mathrm{M}$ lyso-PC perfusion of lungs from LPS-pretreated rats $(P<0.05, n=4)$.

Lipid priming of isolated rat neutrophils. Because neutrophils are thought to play a central role in ALI and little infor- 


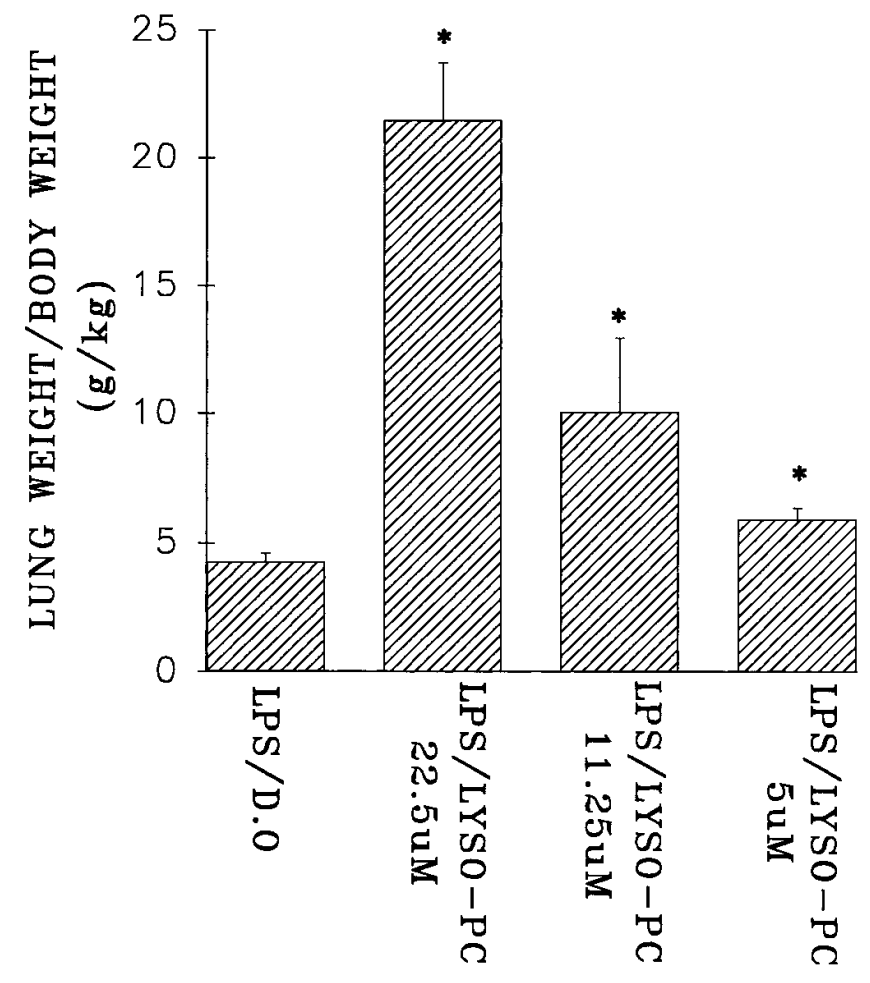

Figure 7. Changes in the ration of lung weight to total body weight $(\mathrm{g} / \mathrm{kg})$, a measure of pulmonary edema, as a function of lyso-PC concentration in the perfusate as compared with D.0 PRBC plasma perfusion in isolated lungs from LPS-pretreated animals. All doses of lyso-PCs employed caused a significant increase in pulmonary edema as compared with the D.0 PRBC plasma-perfused lungs $(P<0.05$, $n=4$ each group).

mation is available in regard to the effects of priming agents on rat neutrophils, rat neutrophils were isolated, and their ability to produce superoxide anion $\left(\mathrm{O}_{2}^{-}\right)$was studied in vitro $(3,4$, 17, 18). Isolated rat neutrophils were primed with KrebsRinger-phosphate-dextrose buffer, $\mathrm{pH} 7.35$, (buffer), $5 \mu \mathrm{M}$ lyso-PCs, or $2 \mu \mathrm{M}$ PAF for $3 \mathrm{~min}$ at $37^{\circ} \mathrm{C}$. The respiratory burst was initiated with the addition of buffer control, or $1 \mu \mathrm{M}$ fMLP. Neutrophils primed and activated with buffer produced negligible amounts of $\mathrm{O}_{2}^{-}$(Fig. 8.). Neutrophils primed with buffer and activated with fMLP produced a small but significant increase in $\mathrm{O}_{2}{ }^{-}$compared with buffer-treated controls. In contrast, neutrophils primed with lyso-PCs or PAF and stimu-

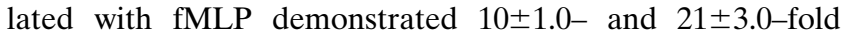
priming of the fMLP-activated respiratory burst, respectively. These results were analogous to the previous data with human neutrophils demonstrating similar in vitro effects of lyso-PCs (25). Thus, rat neutrophils demonstrate a priming response which is qualitatively comparable to that observed in human neutrophils; however, rat neutrophils generate a much smaller amount of $\mathrm{O}_{2}{ }^{-}$in response to stimuli in comparison to previous studies with human neutrophils $(25,33)$.

\section{Discussion}

The pathogenesis of TRALI is not completely understood. Previous publications have focused on the transfusion of im- munoglobulins directed against recipient leukocytes resulting in pulmonary leukostasis and subsequent activation of these neutrophils resulting in ALI. However, many of these reactions do not involve specific HLA or granulocyte antibodies of donor origin $(20,22)$. Moreover, in an ex vivo rabbit lung model of TRALI immune-mediated ALI could only be produced if plasma containing neutrophil antibodies with welldefined antigen specificity was mixed with neutrophils that expressed this specific antigen and then perfused. Conversely, if plasma containing this specific neutrophil antibody was mixed with neutrophils that did not express this specific antigen and perfused, TRALI was not produced (34). The data presented provide another etiology for TRALI by demonstrating that biologically active lipids, produced during blood storage, cause ALI in a two-step animal model of pulmonary injury. These data also serve as a bridge between the clinical descriptions of TRALI (19-22) and both the two insult animal models of ALI $(17,18)$ and the observed effects of these lipids on neutrophil activity in vitro $(9-16,25,33)$. One element of the model is infection, represented by the use of endotoxin. LPS was employed because infection has been identified as a patient risk factor associated with TRALI (22). The second insult, the infusion of the plasma fraction of stored PRBCs, is based upon the in vitro description of the generation of biologically active compounds during routine storage of cellular blood components and the effects of these compounds on human neutrophils (25). This novel animal model of TRALI has the potential to aid in our understanding of the diverse etiologic factors involved with TRALI and ALI as a whole.

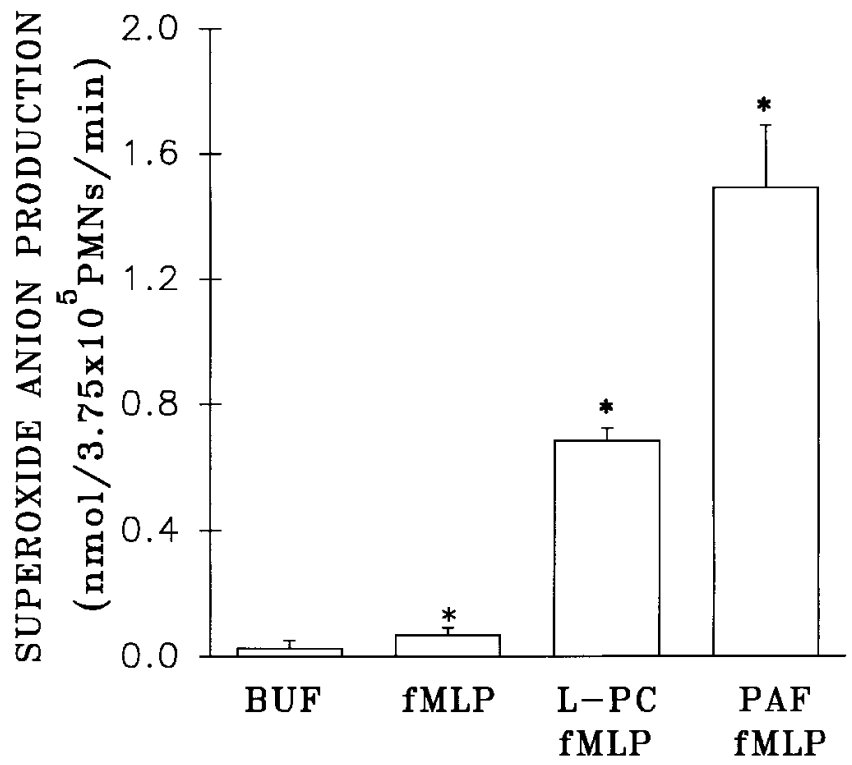

Figure 8. The maximal rate of superoxide anion production as a function of treatment group in neutrophils isolated from healthy rats. Formyl-Met-Leu-Phe $(f M L P, 1 \mu \mathrm{M})$ caused a significant increase in superoxide anion production in buffer pretreated rat PMNs as compared with buffer-pretreated, buffer-stimulated controls $(P<0.05)$. Both platelet-activating factor $(P A F)$ and lyso-PC-primed PMNs, stimulated with fMLP demonstrated a significant augmentation of superoxide anion production as compared with the buffer-treated PMNs and those PMNs stimulated with fMLP alone $(P<0.05, n=4$ for each group). 
The important new information obtained from this work is that plasma from stored blood, but not fresh blood, causes lung injury in lungs from rats pretreated with endotoxin. The plasma fraction of D.42 PRBCs in lungs from LPS-pretreated rats caused vasoconstriction and edema formation, both of which were inhibited by preincubation with a PAF receptor antagonist or a calcium-entry blocker. Lipid extracts from D.42 PRBC plasma and the purified lipids from stored PRBCs, lyso-PCs, caused little vasoconstriction in the lungs from LPS-pretreated rats, except at the highest dose of lysoPCs employed. Both the plasma lipid extract and lyso-PCs elicited severe lung edema in the lungs from LPS-pretreated animals. The concentrations of lyso-PCs employed (4.5, 11.25, and $22.5 \mu \mathrm{M}$ ) correspond to the amounts of lyso-PCs infused with the administration of 2,4 , and $8 \mathrm{U}$ of PRBCs, respectively to an average $70 \mathrm{~kg}$ adult.

The mechanism of acute lung injury in this model relates to the effects of LPS pretreatment on neutrophil-endothelial cell interactions. LPS is known to prime neutrophils, a process that augments the respiratory burst and the release of granule contents in response to a subsequent stimulus $(10,35)$. Exposure to LPS in vitro directly causes increased cell surface expression of adhesion molecules on the neutrophil including the $\beta_{2}$-integrins which allow for tight adherence to endothelium and the extracellular matrix (36). Not only neutrophils are affected by LPS, for LPS incubation in vitro activates vascular endothelium causing direct increases in the cell surface expression of adhesion molecules including the selectins and intracellular adhesion molecule $1(15,16,37,38)$. Selectins are important for neutrophil tethering or rolling along the surface of endothelium, whereas intracellular adhesion molecule 1 is the ligand for the neutrophil $\beta_{2}$-integrins involved in the firm adhesion of neutrophils to endothelium $(15,16,37-40)$. It seems plausible that after LPS pretreatment, these mechanisms are involved in the pulmonary sequestration of neutrophils, as demonstrated by the histology, via tethering and firm adhesion of these granulocytes to activated pulmonary endothelium (35-40). A second insult, in our model the perfusion of the lungs with plasma from stored blood, could activate the adherent neutrophils and cause the release of neutrophil granule contents, active oxygen species and a number of lipid mediators $(1,3,4,13,17,18,22$, 39). Previous work using this identical lung model has demonstrated that the recruitment of inflammatory cells, i.e., PMNs are essential for ALI and especially for the generation of leukotrienes and other markers of pulmonary damage (41). Moreover, because leukotrienes have been demonstrated to be directly involved in ALI and LTE4 is the final breakdown product of this biologic cascade, the increased levels of LTE4 in the perfusate after day 42 PRBC plasma perfusion of lungs from LPS-pretreated animals is consistent with pulmonary cell damage and ALI (41-45).

PAF receptor blockade and inhibition of calcium entry were both effective in reducing the pulmonary vasoconstriction and lung edema formation after addition of the D.42 PRBC plasma to the lungs from LPS-pretreated animals. Since LPS had been given to the animals before lung isolation, it is clear that these agents did not interfere with neutrophil recruitment to the lung vasculature as further documented by the histology. Instead, it is likely that theses inhibitors blocked the further activation of the neutrophil in situ, the action of factors released by the neutrophil, or both. The PAF receptor antagonist WEB 2170 blocks the effects of lyso-PCs on neutro- phils in vitro and such inhibition of PMN function may decrease the amount of lung injury in this model. Leukotriene release is an indication of ALI; therefore, we interpret the increased amounts of LTE4 as a marker of pulmonary injury (42). However, PAF receptor antagonism was not as effective in blocking pulmonary edema $(29 \pm 10 \%, P<0.05)$ in response to the highest lyso-PC concentrations $(22.5 \mu \mathrm{M})$. WEB 2170 is a competitive antagonist, and these higher concentrations may possibly overwhelm the concentration of inhibitor employed in these experiments (28). For example, to maximize the effects of lyso-PCs and PAF on human neutrophils in vitro WEB 2170 concentrations need to reach $400 \mu \mathrm{M}$, virtually an order of magnitude higher that used in these experiments (25). Similar to PAF receptor blockade, calcium channel antagonism also inhibited lung injury in this model. The calcium antagonist mibefradil likely has many targets, especially the verapamil and diltiazem inhibitable $\mathrm{Ca}^{2+}$ channels on the endothelium which are also sensitive to mibefradil $(31,32,46)$. Although neutrophils are less likely to be effected by mibefradil because they do not possess voltage gated or L-type $\mathrm{Ca}^{2+}$ channels, both verapamil and nifedipine, potent L-type $\mathrm{Ca}^{2+}$ channel antagonists, inhibit the respiratory burst and bactericidal activity of neutrophils in vitro (47-50). Nevertheless, mibefradil was effective in reducing $\mathrm{PMN}$-mediated lung injury, and the mechanism of these effects through $\mathrm{Ca}^{2+}$ channel blockade requires further elucidation.

The observed lung injury was not due to immunologic incompatibilities because fresh human plasma and human plasma from fresh blood, D.0 PRBCs, did not cause immunologic based injury whether the rats were pretreated with LPS or saline control. Moreover, many of the D.42 PRBC plasma fractions used for these experiments were paired samples so that both the D.0 and D.42 plasma samples came from the identical units. In addition, the lipid extracts from D.42 PRBC plasma or purified lipids of synthetic origin do not include immunoglobulins or the cellular elements that would account for a possible immune mechanism in the genesis of acute lung injury. However, since there was clearly a different response profile between the D.42 PRBC plasma and the lipid extract, it is possible that the D.42 PBRC plasma contained additional active compounds which contribute to the vasoconstrictive response. Moreover, these substances and their vasoconstrictive effects were eliminated by lipid extraction. These additional compounds might include cytokines which have been shown to be generated during routine storage of both PRBCs and platelet concentrates separated from whole blood (51-54).

This study has documented that the plasma fraction of older stored PRBCs (D.42) but not the plasma from fresh PRBCs (D.0) is etiologic in this animal model of TRALI. These results correlate well with clinical information for TRALI and other adverse events of transfusion. A retrospective study of TRALI from this laboratory suggested that older components, both PRBCs and platelet concentrates, may be associated with TRALI (22). Moreover, recently we have reported a nested case control study of 72 patients with TRALI compared with 225 concurrently transfused control patients which has confirmed that TRALI is linked to the transfusion of older, cellular components $(22,55)$. In addition, longer storage times of platelet concentrates also correlate with increased levels of cytokines and lipids in the platelet products and with an increased prevalence of transfusion reactions as a whole $(33,51-$ $54,56)$. 
In conclusion, lyso-PCs from stored packed red blood cells played an etiologic role in this animal model of ALI. Acute lung injury after transfusion, TRALI, involves a number of complex cellular processes including activation of endothelium, recruitment and adherence of neutrophils (resulting in granulocyte sequestration), and activation of these adherent neutrophils leading to endothelial injury, capillary leak, and pulmonary damage. More than one agent is required to orchestrate the complicated interactions of neutrophils with vascular endothelium as demonstrated by endotoxin-induced pulmonary recruitment of neutrophils and activation of these neutrophils by factors contained in the plasma fraction of stored PRBCs, especially lyso-PCs. The studies presented here have focused on lyso-PCs because of their presence in the plasma from patients with TRALI $(22,55)$. Other agents such as cytokines, leukocyte specific antibodies, and complement may also contribute to the sequence of events resulting in lung injury $(19,20,34,52-54,56)$. The data presented here has demonstrated that TRALI may be precipitated by two sequential insults, one consisting of the clinical condition of the transfusion recipient and the other due to the transfusion of factors in the plasma fraction of older, stored blood components. Thus, TRALI, like ARDS, appears to be the result of at least two insults which effect the interactions of the pulmonary endothelium with the circulating neutrophil.

\section{Acknowledgments}

The authors would like to acknowledge Patrick H. Murphy from the Department of Information Services at Bonfils Blood Center for preparation of figures in this manuscript.

This work was supported by a Clinical Associate Physician Award (M01-RR00069) from the General Clinical Research Centers Program, National Centers for Research Resources, National Institutes of Health, a grant from the National Blood Foundation (C.C. Silliman), a grant-in-aid from the American Heart Association, a R01 grant (HL43180) and a Vascular Academic Award from NIH (N.F. Voelkel), a Transfusion Medicine Academic Award (K07-HL02036) from the National Heart, Lung, and Blood Institute, NIH, and the Stacy Marie True Memorial Trust (D.R. Ambruso), and the Bonfils Blood Center.

\section{References}

1. Weiss, S.J. 1989. Tissue destruction by neutrophils. N. Engl. J. Med. 320: $365-376$

2. Sperling, R.J., A.I. Benincasco, R.J. Anderson, J.S. Coblyn, K.F. Austen, and M.E. Weinblatt. 1992. Acute and chronic suppression of leukotriene B4 synthesis ex vivo in neutrophils from patients with rheumatoid arthritis beginning methotrexate. Arthritis Rheum. 35:376-384.

3. Boxer, L.A., R. Axtell, and S. Suchard. 1990. The role of the neutrophil in inflammatory diseases of the lung. Blood Cells (NY). 16:25-42.

4. Repine, J.E. 1992. Scientific perspectives on ARDS. Lancet (N. Am. Ed.). 339:466-469.

5. Rinaldo, R.E., and R.M. Rogers. 1982. Adult respiratory syndrome: changing concepts of lung injury and repair. N. Engl. J. Med. 306:900-909.

6. Zimmerman, G.A., A.D. Renzetti, and H.R. Hill. 1983. Functional and metabolic activity of granulocytes from patients with the adult respiratory distress syndrome: evidence for activated neutrophils in the pulmonary circulation. Am. Rev. Respir. Dis. 127:290-300.

7. Parsons, P.E., A.A. Fowler, T.M. Hyers, and P.M. Henson. 1985. Chemotactic activity in bronchoalveolar lavage fluid from patients with the adult respiratory distress syndrome. Am. Rev. Respir. Dis. 132:490-493.

8. Rinaldo, J.E., and H. Borovetz. 1985. Deterioration of oxygenation and abnormal lung microvascular permeability during resolution of leukopenia in patients with diffuse lung injury. Am. Rev. Respir. Dis. 131:579-583.

9. Ingraham, L.M., T.D. Coates, J.M. Allen, C.P. Higgins, R.L. Baehner, and L.A. Boxer. 1982. Metabolic, membrane, and functional responses of hu- man polymorphonuclear leukocytes to platelet activating factor. Blood. 59: 1259-1266.

10. McPhail, L.C., C.C. Clayton, and R. Snyderman. 1984. The NADPH oxidase of human polymorphonuclear leukocytes: evidence for regulation by multiple signals. J. Biol. Chem. 259:5768-5775.

11. Guthrie, L.A., L.C. McPhail, P.M. Henson, and R.B. Johnston, Jr. 1984 The priming of neutrophils for enhanced release of oxygen metabolites by bacterial lipopolysaccharide: evidence for increased activity of the superoxide producing enzyme. J. Exp. Med. 160:1656-1671.

12. Ingraham, L., J.E. Lafuze, L.A. Boxer, and R.L. Baehner. 1987. In vitro and in vivo effects of treatment by platelet activating factor on $N$-formyl-metleu-phe mediated responses of polymorphonuclear leukocytes. Br. J. Haematol. 66:219-225.

13. Vercellotti, G.M., H.Q. Yin, K.S. Gustafson, R.D. Nelson, and H.S. Jacob. 1988. Platelet activating factor primes neutrophil responses to agonists: role in promoting neutrophil-mediated endothelial damage. Blood. 71:1100 1107.

14. Detmers, P.A., S.K. Lo, E. Olson-Eggbert, A. Walz, M. Baggiolini, and Z.A. Cohn. 1990. Neutrophil activating protein-1/interleukin-8 stimulates the binding activity of the leukocyte adhesion receptor CD11b/CD18 on human neutrophils. J. Exp. Med. 171:1155-1162.

15. Albelda, S.M., C.W. Smith, and P.A. Ward. 1994. Adhesion molecules and inflammatory injury. FASEB J. 8:504-512.

16. Granger, D.N., and P. Kubes. 1994. The microcirculation and inflammation: modulation of leukocyte-endothelial cell adhesion. J. Leukocyte Biol. 55: $662-675$.

17. Salzer, W.L., and C.E. McCall. 1990. Primed stimulation of isolated perfused rabbit lung by endotoxin and platelet activating factor induces enhanced production of thromboxane and lung injury. J. Clin. Invest. 85:1135-1143.

18. Rabinovici, R., K.M. Esser, P.G. Lysko, T.-L. Yue, D.E. Griswold, L.M. Hillegass, P.J. Bugelski, J.M. Hallenbeck, and G. Feuerstein. 1991. Priming by platelet-activating factor of endotoxin-induced lung injury and cardiovascular shock. Circ. Res. 69:12-25.

19. Popovsky, M.A., and S.B. Moore. 1985. Diagnostic and pathogenetic considerations in transfusion-related acute lung injury. Transfusion (Bethesda). 25:573-577.

20. Popovsky, M.A., and H.C. Chapin. 1993. Transfusion related acute lung injury. Transfusion (Bethesda). 33:444-445.

21. Kolleff, M.H., and D.P. Schuster. 1995. The acute respiratory distress syndrome. N. Engl. J. Med. 332:27-37.

22. Silliman, C.C., A.J. Paterson, W.O. Dickey, D.F. Stroncek, M.A. Popovsky, S.A. Caldwell, and D.R. Ambruso. 1997. The association of biologically active lipids with the development of transfusion-related acute lung injury: a retrospective study. Transfusion (Bethesda). 37:719-726.

23. Walker, R.H. 1993. American Association of Blood Banks: Technical Manual, 11th edition. American Association of Blood Banks. Bethesda, MD. 51-74.

24. Parent, R. 1992. Isolated perfused lung preparation as a research tool. In Comparative Biology of the Lung. S.W. Chang and N.F. Voelkel, editors. CRC Press, Inc., Boca Raton, FL. 587-613.

25. Silliman, C.C., K.L. Clay, G.W. Thurman, C.A. Johnson, and D.R. Ambruso. 1994. Partial characterization of lipids that develop during the routine storage of blood and prime the neutrophil NADPH oxidase. J. Lab. Clin. Med. 124:684-694.

26. Chang, S., J.Y. Westcott, W.C. Pickett, R.C. Murphy, and N.F. Voelkel. 1989. Endotoxin induced lung injury in rats: the role of eicosanoids. J. Appl. Physiol. 66:2407-2418.

27. Sugawara, T., M. Miyamoto, S. Takayama, and M. Kato. 1995. Separation of neutrophils from blood in human and laboratory animals and comparison of the chemotaxis. J. Pharmacol. Toxicol. Methods. 33:91-100.

28. Heuer, H.O., J. Casals-Stenzel, G. Muacevic, and K.H. Weber. 1990. Pharmacologic activity of bepafant (WEB 2170), a new and selective hetrazapinoic antagonist of platelet activating factor. J. Pharmacol. Exp. Ther. 255:962968

29. Ono, S., J.Y. Westcott, and N.F. Voelkel. 1992. PAF antagonists inhibit pulmonary vascular remodeling induced by hypobaric hypoxia in rats. J. Appl. Physiol. 73:1084-1092.

30. Ono, S., and N.F. Voelkel. 1991. PAF antagonists inhibit monocrotaline-induced lung injury and pulmonary hypertension. J. Appl. Physiol. 71: 2483-2492.

31. Veniant, M., J.-P. Clozel, P. Hess, and R. Wolfgang. 1991. Ro 40-5967, in contrast to diltiazem, does not reduce left ventricular contractility in rats with chronic myocardial infarction. J. Cardiovasc. Pharmacol. 17:277-284.

32. Osterrieder, W., and M. Holck. 1989. In vitro pharmacologic profile of Ro 40-5967, a novel $\mathrm{Ca}^{2+}$ channel blocker with potent vasodilator but weak inotropic action. J. Cardiovasc. Pharmacol. 13:754-759.

33. Silliman, C.C., W.O. Dickey, A.J. Paterson, G.W. Thurman, K.L. Clay, C.A. Johnson, and D.R. Ambruso. 1996. Analysis of the priming activity of lipids generated during routine storage of platelet concentrates. Transfusion (Bethesda). 36:133-139.

34. Seeger, W., U. Schneider, and B. Kreusler. 1990. Reproduction of transfusion related acute lung injury in an ex vivo lung model. Blood. 76:1438-1444.

35. Fittschen, C., R.A. Sandhaus, G.S. Worthen, and P.M. Henson. 1988. 
Bacterial lipopolysaccharide enhances chemoattractant elastase secretion by human neutrophils. J. Leukocyte Biol. 43:547-556.

36. Lynam, E.B., S.I. Simon, Y.P. Rochon, and L.A. Sklar. 1994. Lipopolysaccharide enhances CD11b/CD18 function but inhibits neutrophil aggregation. Blood. 83:3303-3311.

37. Bevilacqua, M.P., and R.M. Nelson. 1993. Selectins. J. Clin. Invest. 91: 379-387.

38. Schumann, R.R., D. Pfeil, N. Lamping, C. Kirschning, G. Scherzinger, P. Sclag, L. Karawajew, and F. Herrmenn. 1996. Lipopolysaccharide induces the rapid tyrosine phosphorylation of the mitogen activated protein kinase erk-1 and p38 in cultured human vascular endothelial cells. Blood. 87:2805-2814.

39. Ford-Hutchinson, A.W., M.A. Bray, M.V. Doig, M.E. Shipley, and M.J. Smith. 1980. Leukotriene B4, a potent chemokinetic and aggregating substance released from polymorphonuclear leukocytes. Nature. 286:264-265.

40. Tonnesen, M.G., D.C. Anderson, T.A. Springer, A. Knedler, N. Avdi, and P.M. Henson. 1989. Adherence of neutrophils to cultured human microvascular endothelial cells. Stimulation by chemotactic peptides and lipid mediators and dependence upon the Mac-1, LFA-1, p150,95, glycoprotein family. J. Clin. Invest. 83:637-646.

41. Voelkel, N.F., J. Czartolomna, J. Simpson, and R.C. Murphy. 1992. FMLP causes eicosanoid-dependent vasoconstriction and edema in lungs from LPS primed rats. Am. Rev. Respir. Dis. 145:701-711.

42. Westcott, J.Y., K. Johnston, R.A. Batt, S.E. Wenzel, and N.F. Voelkel. 1990. Measurement of peptidoleukotrienes in biological fluids. J. Appl. Physiol. 68:2640-2648.

43. Chang, S.-W., J. Czartolomna, and N.F. Voelkel. 1992. Role of eicosanoids in staphylococcal $\alpha$-toxin-induced lung injury in the rat. Am. J. Physiol. 262: L502-510.

44. Maycock, L.A., S.S. Pong, J.F. Evans, and D.K. Miller. 1989. Biochemistry of the lipoxygenase pathways. In Leukotrienes and Lipoxygenases. Elsevier Science, Inc., New York. 1-599.

45. Dahlen, S.E., P. Hedqvist, S. Hammarstrom, and B. Samuelsson. 1980. Leukotrienes are potent constrictors of human bronchi. Nature. 288:484-486.
46. Ono, S., and N.F. Voelkel. 1992. Inflammation and pulmonary hypertension during hypoxia. In High Altitude Medicine. G. Ueda, editor. Shinsu University Press, Matsumoto, Japan. 347-654.

47. Krause, K.H., and M.J. Welsh. 1990. Voltage dependent and $\mathrm{Ca}^{2+}$ activated ion channels in human neutrophils. J. Clin. Invest. 85:491-498.

48. Schrenzel, J., N. Demaurex, D.P. Lew, and K.H. Krause. 1995. Characterization of $\mathrm{Ca}^{2+}$ influx in human neutrophils using the patch clamp technique. Schweiz. Med. Wochenschr. 125:1174-1178.

49. Zimmerman, J.J., S.M. Zuk, and J.R. Millard. 1988. In vitro modulation of human neutrophil superoxide anion generation by various calcium channel antagonists used in ischemia-reperfusion resuscitation. Biochem. Pharmacol. 38:3601-3610.

50. Kazanjian, P.H., and J.E. Pennington. 1985. Influence of drugs that block calcium channels on the microbicidal function of human neutrophils. $J$. Infect. Dis. 15:15-22.

51. Shanwell, A., M. Kristiansson, M. Remberger, and O. Ringden. 1997. Generation of cytokines in red cell concentrates during storage is prevented by prestorage white cell reduction. Transfusion (Bethesda). 37:678-684.

52. Muylle, L., M. Joos, E. Wouters, R. DeBock, and M.E. Peetermans. 1993. Increased tumor necrosis factor $\alpha(\mathrm{TNF} \alpha)$, interleukin 1 , and interleukin 6 levels in the plasma of stored platelet concentrates: relationship between TNF $\alpha$ and IL-6 levels and febrile transfusion reactions. Transfusion (Bethesda). 33:195-199.

53. Stack, G., and E.L. Snyder. 1994. Cytokine generation in stored platelet concentrates. Transfusion (Bethesda). 34:20-25.

54. Heddle, N.M., L. Klama, J. Singer, C. Richards, P. Fedak. I. Walker, J.G. Kelton. 1994. The role of plasma from platelet concentrates in transfusion reactions. N. Engl. J. Med. 331:625-628.

55. Boshkov, L., C. Silliman, G. Clarke, W. Dickey, and D. Ambruso. 1995. Transfusion related acute lung injury following platelet transfusion: a study of possible etiologic factors. Blood. 86(Suppl.):354a.

56. Muylle, L., E. Wouters, R. De Bock, and M.E. Peetermans. 1992. Reactions to platelet transfusion: the effect of the storage time of the concentrate. Transfus. Med. 2:289-293. 